\title{
Impact of nuclear data evaluations on data assimilation for an LFR
}

\author{
Pablo Romojaro ${ }^{1, *}$ and Francisco Álvarez-Velarde ${ }^{1, * *}$ \\ ${ }^{1}$ Centro de investigaciones Energéticas, Medioambientales y Tecnológicas (CIEMAT), Energy Department, Avda. Complutense 40, \\ 28040 Madrid, Spain
}

\begin{abstract}
The Lead-cooled Fast Reactor is one of the three technologies selected by the Sustainable Nuclear Energy Technology Platform that can meet future European energy needs. The main drawbacks for the industrial deployment of LFR are the lack of operational experience and the impact of uncertainties. In nuclear reactor design the uncertainties mainly come from material properties, fabrication tolerances, operation conditions, simulation tools and nuclear data. The uncertainty in nuclear data is one of the most important sources of uncertainty in reactor physics simulations. Furthermore, it is known that the uncertainties in reactor criticality safety parameters are severely dependent on the nuclear data library used to estimate them. However, the impact of using different evaluations while performing data assimilation to constraint the uncertainties in the criticality parameters has not been properly assessed yet. In this work, a data assimilation for the main isotopes contributing to the uncertainty in $k_{\text {eff }}$ of the ALFRED lead-cooled fast reactor has been performed with the SUMMON system using JEFF-3.3, ENDF/B-VIII.0 and JENDL-4.0u2 state-of-the-art nuclear data libraries, together with critical mass experiments from the International Criticality Safety Benchmark Evaluation Project that are representative of ALFRED, in order to assess the impact of using different evaluations for data assimilation.
\end{abstract}

\section{Introduction}

The Gen-IV International Forum [1] has identified and selected six nuclear energy systems for further research and development, that can help meet the world's future energy needs. In parallel, Europe with the European Sustainable Nuclear Industrial Initiative [2] has chosen three Gen-IV fast reactor technologies as candidates that can satisfy future European energy demands: the sodium-cooled fast reactors (SFR), the lead-cooled fast reactors (LFR) and the gas-cooled fast reactors (GFR). However, there are some drawbacks that need to be solved for the industrial deployment of LFR. The main drawbacks are the lack of operational experience and the impact of uncertainties. In nuclear reactor design the uncertainties mainly come from material properties, fabrication tolerances, operation conditions, simulation tools and nuclear data. For reactor physics simulations, the uncertainty in nuclear data plays a key role and is one of the most important sources of uncertainty [3] in the neutronic and safety assessment of a new reactor design. However, significant gaps between the current uncertainties and the target accuracies have been systematically shown in the past [4]. Meeting the target accuracy is required to achieve the requested level of safety for innovative reactor designs and to minimize the increase in the costs due to additional security measures. In particular, for fast reactors, the target accuracy defined for the effective neutron multiplication factor is $300 \mathrm{pcm}$, as stablished in 2008 by the SG-26 of the Work-

\footnotetext{
*e-mail: pablo.romojaro@ciemat.es

**e-mail: francisco.alvarez@ ciemat.es
}

ing Party on International Nuclear Data Evaluation Cooperation of OECD/NEA [5]. Furthermore, it is known that the uncertainties in reactor criticality safety parameters are severely dependent on the nuclear data library used to estimate them. Therefore, the objective of this work is to quantify the uncertainty in $k_{\text {eff }}$ for a reference LFR due to uncertainties in nuclear data using state-of-the-art libraries and, if target accuracies are not met, perform assimilation on the major contributors in order to constrain the uncertainties in a new library created specifically for LFR applications. Several LFR concepts are now in design phase in Europe, such as the Multi-purpose hYbrid Research Reactor for High-tech Applications (MYRRHA) [6] and the Advanced Lead-cooled Fast Reactor European Demonstrator (ALFRED) [7]. ALFRED has been taken as the reference design for the analyses. ALFRED is a 300 MWth small-size pool type reactor cooled by lead, with the core, primary pumps and steam generators contained within the reactor vessel. It shall be connected to the electrical grid (125 MWe) and use already available technology to speed up construction time, with corrosion-resistant structural materials compatible with lead. ALFRED core is composed by 171 hexagonal fuel assemblies divided in an inner and an outer zone, with different plutonium enrichments (higher enrichment in the outer region) to flatten the power distribution (Fig. 1).

JEFF-3.3 [8], ENDF/B-VIII.0 [9] and JENDL-4.0u2 [10] processed with NJOY2016 [11] have been used. The uncertainty quantification has been carried out with the SUMMON code $[12,13]$, which has been developed by 




Figure 1. ALFRED core model.

Table 1. $k_{\text {eff } f}$ uncertainty quantification for ALFRED with JEFF-3.3.

\begin{tabular}{clllc}
\hline \multicolumn{3}{c}{ Quantity } & $\Delta k_{\text {eff }} / k_{\text {eff }}(\%)$ \\
\hline${ }^{240} \mathrm{Pu}$ & $(\mathrm{n}, \mathrm{f})$ & ${ }^{240} \mathrm{Pu}$ & $(\mathrm{n}, \mathrm{f})$ & 0.572 \\
${ }^{240} \mathrm{Pu}$ & $(\mathrm{n}, \mathrm{f})$ & ${ }^{240} \mathrm{Pu}$ & $(\mathrm{n}, \gamma)$ & -0.441 \\
${ }^{239} \mathrm{Pu}$ & $v_{\mathrm{p}}$ & ${ }^{239} \mathrm{Pu}$ & $v_{\mathrm{p}}$ & 0.319 \\
${ }^{239} \mathrm{Pu}$ & $(\mathrm{n}, \mathrm{f})$ & ${ }^{239} \mathrm{Pu}$ & $(\mathrm{n}, \mathrm{f})$ & 0.307 \\
${ }^{239} \mathrm{Pu}$ & $\chi$ & ${ }^{239} \mathrm{Pu}$ & $\chi$ & 0.306 \\
${ }^{238} \mathrm{U}$ & $(\mathrm{n}, \mathrm{n})$ & ${ }^{238} \mathrm{U}$ & $(\mathrm{n}, \mathrm{n})$ & 0.254 \\
${ }^{238} \mathrm{U}$ & $(\mathrm{n}, \gamma)$ & ${ }^{238} \mathrm{U}$ & $(\mathrm{n}, \gamma)$ & 0.224 \\
${ }^{240} \mathrm{Pu}$ & $(\mathrm{n}, \gamma)$ & ${ }^{240} \mathrm{Pu}$ & $(\mathrm{n}, \gamma)$ & 0.207 \\
${ }^{238} \mathrm{U}$ & $(\mathrm{n}, \mathrm{n})$ & ${ }^{238} \mathrm{U}$ & $(\mathrm{n}, \mathrm{f})$ & -0.205 \\
${ }^{239} \mathrm{Pu}$ & $(\mathrm{n}, \mathrm{f})$ & ${ }^{239} \mathrm{Pu}$ & $(\mathrm{n}, \gamma)$ & 0.185 \\
\multicolumn{7}{c}{ Total uncertainty in $k_{\text {eff }}$} & 0.756 \\
\hline \multicolumn{4}{c}{}
\end{tabular}

CIEMAT to perform complete automated sensitivity and uncertainty analyses of the most relevant criticality safety parameters of detailed complex reactor designs from the neutronic point of view, i.e., $k_{e f f}, \beta_{e f f}, \Lambda_{e f f}$ and reactivity coefficients, using state-of-the-art nuclear data libraries and covariances. The data assimilation (DA) has been done with DAWN [14], a data adjustment code developed by CIEMAT that uses Bayesian inference and a deterministic approach (the generalized least squares methodology) and integral experiments from the International Criticality Safety Benchmark Evaluation Project (ICSBEP) similar to the system, to adjust nuclear data and constrain uncertainties. Finally, a model of ALFRED at nominal conditions at Beginning of Cycle has been used in the calculations.

\section{Uncertainty quantification}

Results from the uncertainty analyses are presented in Table 1 for JEFF-3.3, Table 2 for ENDF/B-VIII.0 and Table 3 for JENDL-4.0u2.

It can be seen that JEFF-3.3 and ENDF/B-VIII.0 are in good agreement, even though the contributors to the uncertainty are different or have different order of magnitude. On the other hand, JENDL-4.0u2 predicts lower uncertainty than the other two evaluations, while also having
Table 2. $k_{\text {eff }}$ uncertainty quantification for ALFRED with ENDF/B-VIII.0.

\begin{tabular}{|c|c|c|c|c|}
\hline \multicolumn{4}{|c|}{ Quantity } & $f f / k_{e f f}(\%)$ \\
\hline${ }^{239} \mathrm{Pu}$ & $(\mathrm{n}, \mathrm{f})$ & ${ }^{239} \mathrm{Pu}$ & $(\mathrm{n}, \mathrm{f})$ & 0.577 \\
\hline${ }^{239} \mathrm{Pu}$ & $(\mathrm{n}, \gamma)$ & ${ }^{239} \mathrm{Pu}$ & $(\mathrm{n}, \gamma)$ & 0.214 \\
\hline${ }^{239} \mathrm{Pu}$ & $v_{\mathrm{p}}$ & ${ }^{239} \mathrm{Pu}$ & $v_{\mathrm{p}}$ & 0.187 \\
\hline${ }^{238} \mathrm{U}$ & $(\mathrm{n}, \gamma)$ & ${ }^{238} \mathrm{U}$ & $(\mathrm{n}, \gamma)$ & 0.186 \\
\hline${ }^{239} \mathrm{Pu}$ & $\chi$ & ${ }^{239} \mathrm{Pu}$ & $\chi$ & 0.153 \\
\hline${ }^{238} \mathrm{U}$ & $v_{\mathrm{T}}$ & ${ }^{238} \mathrm{U}$ & $v_{\mathrm{p}}$ & 0.151 \\
\hline${ }^{238} \mathrm{U}$ & $\left(\mathrm{n}, \mathrm{n}^{\prime}\right)$ & ${ }^{238} \mathrm{U}$ & $(\mathrm{n}, \mathrm{f})$ & -0.142 \\
\hline${ }^{238} \mathrm{U}$ & $\left(n, n^{\prime}\right)$ & ${ }^{238} \mathrm{U}$ & $\left(\mathrm{n}, \mathrm{n}^{\prime}\right)$ & 0.130 \\
\hline${ }^{238} \mathrm{U}$ & $\left(\mathrm{n}, \mathrm{n}{ }^{\prime}\right)$ & ${ }^{238} \mathrm{U}$ & $(\mathrm{n}, \gamma)$ & 0.127 \\
\hline${ }^{238} \mathrm{U}$ & $v_{\mathrm{T}}$ & ${ }^{238} \mathrm{U}$ & $v_{\mathrm{p}}$ & 0.107 \\
\hline \multicolumn{4}{|c|}{ Total uncertainty in $k_{e f f}$} & 0.720 \\
\hline
\end{tabular}

Table 3. $k_{\text {eff }}$ uncertainty quantification for ALFRED with JENDL-4.0u2.

\begin{tabular}{clllc}
\hline \multicolumn{3}{c}{ Quantity } & $\Delta k_{\text {eff }} / k_{\text {eff }}(\%)$ \\
\hline${ }^{238} \mathrm{U}$ & $\left(\mathrm{n}, \mathrm{n}^{\prime}\right)$ & ${ }^{238} \mathrm{U}$ & $\left(\mathrm{n}, \mathrm{n}^{\prime}\right)$ & 0.306 \\
${ }^{239} \mathrm{Pu}$ & $(\mathrm{n}, \mathrm{f})$ & ${ }^{239} \mathrm{Pu}$ & $(\mathrm{n}, \mathrm{f})$ & 0.268 \\
${ }^{238} \mathrm{U}$ & $(\mathrm{n}, \gamma)$ & ${ }^{238} \mathrm{U}$ & $(\mathrm{n}, \gamma)$ & 0.241 \\
${ }^{239} \mathrm{Pu}$ & $\chi$ & ${ }^{239} \mathrm{Pu}$ & $\chi$ & 0.233 \\
${ }^{239} \mathrm{Pu}$ & $(\mathrm{n}, \gamma)$ & ${ }^{239} \mathrm{Pu}$ & $(\mathrm{n}, \gamma)$ & 0.171 \\
${ }^{239} \mathrm{Pu}$ & $v_{\mathrm{T}}$ & ${ }^{239} \mathrm{Pu}$ & $v_{\mathrm{p}}$ & 0.167 \\
${ }^{56} \mathrm{Fe}$ & $\left(\mathrm{n}, \mathrm{n}^{\prime}\right)$ & ${ }^{56} \mathrm{Fe}$ & $\left(\mathrm{n}, \mathrm{n}^{\prime}\right)$ & 0.162 \\
${ }^{206} \mathrm{~Pb}$ & $\left(\mathrm{n}, \mathrm{n}^{\prime}\right)$ & ${ }^{206} \mathrm{~Pb}$ & $\left(\mathrm{n}, \mathrm{n}^{\prime}\right)$ & 0.147 \\
${ }^{239} \mathrm{Pu}$ & $v_{\mathrm{p}}$ & ${ }^{239} \mathrm{Pu}$ & $v_{\mathrm{p}}$ & 0.118 \\
${ }^{240} \mathrm{Pu}$ & $(\mathrm{n}, \gamma)$ & ${ }^{240} \mathrm{Pu}$ & $(\mathrm{n}, \gamma)$ & 0.114 \\
$\mathrm{Total}$ uncertainty in $k_{\text {eff }}$ & 0.640 \\
\hline
\end{tabular}

different contributors to the uncertainty. This can be attributed to different cross section uncertainty evaluations, missing and different cross section correlations and covariance evaluations.

Nevertheless, what is clearly seen is that $k_{\text {eff }}$ target accuracy is exceeded approximately by a factor of two for all considered nuclear data evaluations. Therefore, data assimilation was carried out for the main common contributors to $k_{\text {eff }}$ uncertainty in the nuclear data libraries analysed, that is: ${ }^{240} \mathrm{Pu}$ (JEFF-3.3), ${ }^{239} \mathrm{Pu}$ (ENDF/B-VIII.0) and ${ }^{238} \mathrm{U}$ (JENDL-4.0u2).

\section{Data assimilation}

Before nuclear data can be adjusted, the first step is to select a comprehensive set of complementary experiments representative of the target application. In practice, this means calculating correlations among the reactor concept and experiments to determine how representative is the latter of the former. This was done using only integral experiments available in the ICSBEP and selecting benchmarks representative of ALFRED based on the representativity factor, $f_{R E}$, whose mathematical formulation is given in the formula below, where $S$ is the sensitivity vector of an integral parameter (in this case $k_{\text {eff }}$ ) to nuclear data, SR of the targeted reactor and SE of the experiment, and $M_{\sigma}$ is the covariance matrix. 
Table 4. Representativity factor of the experiments used in the assimilation for JEFF-3.3.

\begin{tabular}{lcccc}
\hline$f_{R E}$ & ALFRED & PMF001 & PMF002 & PMF006 \\
\hline ALFRED & 1.00 & 0.71 & 0.88 & 0.52 \\
PMF001 & 0.71 & 1.00 & 0.78 & 0.98 \\
PMF002 & 0.88 & 0.78 & 1.00 & 0.75 \\
PMF006 & 0.52 & 0.98 & 0.75 & 1.00 \\
\hline
\end{tabular}

Table 5. Representativity factor of the experiments used in the assimilation for ENDF/B-VIII.0.

\begin{tabular}{lcccc}
\hline$f_{R E}$ & ALFRED & PMF001 & PMF002 & PMF006 \\
\hline ALFRED & 1.00 & 0.63 & 0.66 & 0.69 \\
PMF001 & 0.63 & 1.00 & 0.97 & 0.93 \\
PMF002 & 0.66 & 0.97 & 1.00 & 0.92 \\
PMF006 & 0.69 & 0.93 & 0.92 & 1.00 \\
\hline
\end{tabular}

$$
f_{R E}=\frac{S_{R}^{T} M_{\sigma} S_{E}}{\sqrt{\left(S_{R}^{T} M_{\sigma} S_{R}\right)\left(S_{E}^{T} M_{\sigma} S_{E}\right)}}
$$

The closer $f_{R E}$ is to one, the more similarity exists between the reactor and the experiment. A set of three experiments were chosen, providing information on separated individual physics effects related to the isotopes with highest sensitivity. However, correlation coefficient data of uncertainties in criticality cases only exist for a limited number of integral experiments. In particular, only for the ones derived from IPPE and from ANL for the ZPR/ZPPR values are publicly available in the ICSBEP database [15]. Therefore, if all three experiments are used in the data adjustment without taking into account correlations, possible compensation effects may occur. Consequently, separate data adjustments have been carried out. The experiments selected were:

- JEZEBEL (PMF001) [16]: a bare sphere of ${ }^{239} \mathrm{Pu}$ metal, sensitive to ${ }^{239} \mathrm{Pu}$;

- ${ }^{240} \mathrm{Pu}$ JEZEBEL (PMF002) [17]: a bare sphere of ${ }^{239} \mathrm{Pu}$ metal with 20.1 at $\%{ }^{240} \mathrm{Pu}$, sensitive to ${ }^{240} \mathrm{Pu}$;

- and PU-MET-FAST-006 (PMF006) [18]: a plutonium sphere reflected by uranium, sensitive to ${ }^{238} \mathrm{U}$.

In Table 4, the representativity factors among ALFRED and the three experiments and among the experiments themselves, calculated with JEFF-3.3 nuclear data library, are shown. Different representativity factors are obtained for the other libraries, as can be seen in Table 5 for ENDF/B-VIII.0.

The experiment closest to ALFRED is ${ }^{240} \mathrm{Pu}$ JEZEBEL for JEFF-3.3, while for ENDF/B-VIII.0 the representativity of the three experiments are nearly the same. There is a close similarity between the experiments, due to all of them employing plutonium as fissile material and having close energy spectrums. Once the DA has been performed with DAWN, the original sensitivity profiles and the posterior covariance matrices were used to propagate the uncertainties.

Using the posterior matrices produced with the assimilation of PMF001, the highest uncertainty reduction (250
Table 6. $k_{\text {eff }}$ uncertainty quantification for ALFRED with posterior ENDF/B-VIII.0 obtained with the DA of PMF001.

\begin{tabular}{ccclc}
\hline \multicolumn{4}{c}{ Quantity } & $\Delta k_{e f f} / k_{e f f}(\%)$ \\
\hline${ }^{239} \mathrm{Pu}$ & $(\mathrm{n}, \mathrm{f})$ & ${ }^{239} \mathrm{Pu}$ & $(\mathrm{n}, \mathrm{f})$ & 0.356 \\
${ }^{239} \mathrm{Pu}$ & $(\mathrm{n}, \gamma)$ & ${ }^{239} \mathrm{Pu}$ & $(\mathrm{n}, \gamma)$ & 0.224 \\
${ }^{239} \mathrm{Pu}$ & $(\mathrm{n}, \mathrm{f})$ & ${ }^{239} \mathrm{Pu}$ & ${ }_{\mathrm{p}}$ & -0.199 \\
${ }^{238} \mathrm{U}$ & $(\mathrm{n}, \gamma)$ & ${ }^{238} \mathrm{U}$ & $(\mathrm{n}, \gamma)$ & 0.186 \\
${ }^{239} \mathrm{Pu}$ & ${ }^{2}$ & ${ }^{239} \mathrm{Pu}$ & $v_{\mathrm{p}}$ & 0.182 \\
$\mathrm{~T}$ & \\
\hline
\end{tabular}

Table 7. $k_{e f f}$ uncertainty quantification for ALFRED with posterior JEFF-3.3 obtained with the DA of PMF001.

\begin{tabular}{|c|c|c|c|}
\hline \multicolumn{3}{|c|}{ Quantity } & $\Delta k_{e f f} / k_{e f f}(\%)$ \\
\hline${ }^{240} \mathrm{Pu}$ & $(\mathrm{n}, \mathrm{f})$ & ${ }^{240} \mathrm{Pu}$ & 0.552 \\
\hline${ }^{240} \mathrm{Pu}$ & $(\mathrm{n}, \mathrm{f})$ & ${ }^{240} \mathrm{Pu}$ & -0.426 \\
\hline${ }^{239} \mathrm{Pu}$ & $(\mathrm{n}, \mathrm{f})$ & ${ }^{239} \mathrm{Pu}$ & 0.303 \\
\hline${ }^{239} \mathrm{Pu}$ & $\chi$ & ${ }^{239} \mathrm{Pu}$ & -0.279 \\
\hline${ }^{239} \mathrm{Pu}$ & $v_{\mathrm{p}}$ & ${ }^{239} \mathrm{Pu} \quad v_{\mathrm{p}}$ & 0.257 \\
\hline \multicolumn{3}{|c|}{ Total uncertainty in $k_{e f f}$} & 0.503 \\
\hline
\end{tabular}

Table 8. $k_{e f f}$ uncertainty quantification for ALFRED with posterior JENDL-4.0u2 obtained with the DA of PMF001.

\begin{tabular}{clllc}
\hline \multicolumn{3}{c}{ Quantity } & & $\Delta k_{\text {eff }} / k_{\text {eff }}(\%)$ \\
\hline${ }^{238} \mathrm{U}$ & $\left(\mathrm{n}, \mathrm{n}^{\prime}\right)$ & ${ }^{238} \mathrm{U}$ & $\left(\mathrm{n}, \mathrm{n}^{\prime}\right)$ & 0.306 \\
${ }^{238} \mathrm{U}$ & $(\mathrm{n}, \gamma)$ & ${ }^{238} \mathrm{U}$ & $(\mathrm{n}, \gamma)$ & 0.241 \\
${ }^{239} \mathrm{Pu}$ & $(\mathrm{n}, \mathrm{f})$ & ${ }^{239} \mathrm{Pu}$ & $(\mathrm{n}, \mathrm{f})$ & 0.239 \\
${ }^{239} \mathrm{Pu}$ & $\chi$ & ${ }^{239} \mathrm{Pu}$ & $\chi$ & 0.215 \\
${ }^{239} \mathrm{Pu}$ & $(\mathrm{n}, \gamma)$ & ${ }^{239} \mathrm{Pu}$ & $(\mathrm{n}, \gamma)$ & 0.170 \\
Total uncertainty in $k_{\text {eff }}$ & 0.590 \\
\hline
\end{tabular}

$\mathrm{pcm}$ ) has been found in ENDF/B-VIII.0 nuclear data library due to the reduction in the contribution of the major contributor, the fission of ${ }^{239} \mathrm{Pu}$ (Table 6).

Moreover, new strong negative cross-correlations between reactions that have large sensitivities appear after the adjustment due to the physical constrains introduced by the experiment, such as the correlation between fission and the prompt neutrons. These negative crosscorrelations also contribute to a significant reduction of the uncertainty. JEFF-3.3 has a similar reduction of uncertainty (Table 7). On the other hand, the assimilation of ${ }^{239} \mathrm{Pu}$ with JENDL only reduces the uncertainty in $50 \mathrm{pcm}$ (Table 8).

The same analyses have been repeated with PMF002 and PMF006. For the assimilation of ${ }^{240} \mathrm{Pu}$ the highest reduction of uncertainty is obtained with JEFF-3.3, with a reduction of nearly $400 \mathrm{pcm}$ using just one integral benchmark and targeting one isotope (Table 9).

On the other hand, assimilation using PMF006 does not have a significant impact in the covariance evaluation and a small reduction of uncertainties is obtained (Table 10).

\section{Conclusions}

An uncertainty quantification of the effective neutron multiplication factor for ALFRED innovative lead cooled fast 
Table 9. $k_{\text {eff }}$ uncertainty quantification for ALFRED with posterior JEFF-3.3 obtained with the DA of PMF002.

\begin{tabular}{|c|c|c|c|}
\hline \multicolumn{3}{|c|}{ Quantity } & $\Delta k_{e f f} / k_{e f f}(\%)$ \\
\hline${ }^{240} \mathrm{Pu}$ & $(\mathrm{n}, \mathrm{f})$ & ${ }^{240} \mathrm{Pu} \quad(\mathrm{n}, \mathrm{f})$ & 0.352 \\
\hline${ }^{240} \mathrm{Pu}$ & $\chi$ & ${ }^{240} \mathrm{Pu}$ & -0.348 \\
\hline${ }^{239} \mathrm{Pu}$ & $v_{\mathrm{p}}$ & ${ }^{240} \mathrm{Pu}$ & -0.308 \\
\hline${ }^{239} \mathrm{Pu}$ & $(\mathrm{n}, \mathrm{f})$ & ${ }^{239} \mathrm{Pu}$ & 0.306 \\
\hline${ }^{239} \mathrm{Pu}$ & $v_{\mathrm{p}}$ & ${ }^{239} \mathrm{Pu} \quad v_{\mathrm{p}}$ & 0.301 \\
\hline \multicolumn{3}{|c|}{ Total uncertainty in $k_{e f f}$} & 0.375 \\
\hline
\end{tabular}

Table 10. $k_{e f f} k_{e f f}$ uncertainty quantification for ALFRED with posterior JENDL-4.0u2 obtained with the DA of PMF006.

\begin{tabular}{cllll}
\hline \multicolumn{3}{c}{ Quantity } & & $\Delta k_{\text {eff }} / k_{\text {eff }}(\%)$ \\
\hline${ }^{238} \mathrm{U}$ & $\left(\mathrm{n}, \mathrm{n}^{\prime}\right)$ & ${ }^{238} \mathrm{U}$ & $\left(\mathrm{n}, \mathrm{n}^{\prime}\right)$ & 0.280 \\
${ }^{239} \mathrm{Pu}$ & $(\mathrm{n}, \mathrm{f})$ & ${ }^{239} \mathrm{Pu}$ & $(\mathrm{n}, \mathrm{f})$ & 0.251 \\
${ }^{238} \mathrm{U}$ & $(\mathrm{n}, \gamma)$ & ${ }^{238} \mathrm{U}$ & $(\mathrm{n}, \gamma)$ & 0.241 \\
${ }^{239} \mathrm{Pu}$ & $\chi$ & ${ }^{239} \mathrm{Pu}$ & $\chi$ & 0.218 \\
${ }^{239} \mathrm{Pu}$ & $(\mathrm{n}, \gamma)$ & ${ }^{239} \mathrm{Pu}$ & $(\mathrm{n}, \gamma)$ & 0.170 \\
Total uncertainty in $k_{\text {eff }}$ & 0.585 \\
\hline
\end{tabular}

reactor has been carried out using the JEFF-3.3, ENDF/BVIII.0 and JENDL-4.0u2 state-of-the-art nuclear data libraries and the SUMMON system. Similar total $k_{\text {eff }}$ uncertainty has been derived using the different libraries, however, the contributors to the uncertainty significantly differ. This has been attributed to differences in the evaluations, to missing correlations and to missing covariance evaluations. Moreover, the uncertainties in $k_{\text {eff }}$ due to uncertainties in nuclear data have been assessed against the target accuracies provided by SG-26 of the WPEC of OECD/NEA in 2008 for LFR. Results show that $k_{\text {eff }}$ target accuracy is exceeded by more than a factor of two using the latest nuclear data evaluations.

In order to constrain the uncertainties in $k_{\text {eff }}$, an assimilation on the main contributors to the uncertainty has been performed using only publicly available critical mass experiments from the International Criticality Safety Benchmark Evaluation Project. A strong dependence of the data assimilation on the nuclear data library used is observed. Furthermore, results show that a reduction of the uncertainty up to 400 pcm can be obtained using just one integral experiment. To reach target accuracies for $k_{\text {eff }}$, a complementary set of integral experiments representative of the system and with high sensitivity to the major contributors to the uncertainty should be used. However, in order to do that, correlations among the experiments are necessary. Therefore, to reach the full potential of the data assimilation technique, it is necessary that the correlations between experiments are evaluated and made available for all users.

\section{References}

[1] Generation IV International Forum, https : //www . gen-4.org/gif/

[2] European Sustainable Nuclear Industrial Initiative, http://www.snetp.eu/esnii/

[3] I. Kodeli, Report OECD/NEA (2017)

[4] H. Harada, A. Plompen, Tech. rep. (2014)

[5] M. Salvatores, G. Aliberti, M. Dunn, A. Hogenbirk, A. Ignatyuk, M. Ishikawa, I. Kodeli, A. Koning, R. McKnight, R. Mills et al., OECD/NEA Nuclear Science NEA/WPEC-26 (2008), ISBN 978-9264-99053-1

[6] G. Van den Eynde, E. Malambu, A. Stankovskiy, R. Fernández, P. Baeten, Journal of Nuclear Science and Technology 52, 1053 (2015)

[7] G. Grasso, C. Petrovich, D. Mattioli, C. Artioli, P. Sciora, D. Gugiu, G. Bandini, E. Bubelis, K. Mikityuk, Nuclear Engineering and Design 278, 287 (2014)

[8] A. Plompen, OECD/NEA JEF/DOC-1864 (2017)

[9] D.A. Brown, M.B. Chadwick, R. Capote, A.C. Kahler, A. Trkov, M.W. Herman, A.A. Sonzogni, Y. Danon, A.D. Carlson, M. Dunn et al., Nuclear Data Sheets 148, 1 (2018)

[10] K. Shibata, O. Iwamoto, T. Nakagawa, N. Iwamoto, A. Ichihara, S. Kunieda, S. Chiba, K. Furutaka, N. Otuka, T. Ohasawa et al., Journal of Nuclear Science and Technology 48, 1 (2011)

[11] A. Kahler, LA-UR-17-20093 (2017)

[12] P. Romojaro, F. Álvarez-Velarde, N. García-Herranz, SUMMON: A Sensitivity And Uncertainty Methodology For MONte Carlo Codes, in Proceedings of International Conference on Mathematics $\mathcal{E}$ Computational Methods Applied to Nuclear Science $\mathcal{E}$ Engineering (MESC 2017) (Jeju, Korea, 2017)

[13] P. Romojaro, F. Álvarez-Velarde, N. García-Herranz, Annals of Nuclear Energy 126, 410 (2019)

[14] P. Romojaro, F. Álvarez-Velarde, N. García-Herranz, EPJ Web of Conferences 211, 05002 (2019)

[15] OECD/NEA, NEA/NSC/DOC(95)03/II (2016)

[16] OECD/NEA, NEA/NSC/DOC(95)03/I Volume I PU-MET-FAST-001 (2016)

[17] OECD/NEA, NEA/NSC/DOC(95)03/I Volume I PU-MET-FAST-002 (2016)

[18] OECD/NEA， NEA/NSC/DOC(95)03/I Volume I PU-MET-FAST-006 (2016) 